\title{
The measurement of total hemoglobin mass (tHb- mass) has a very low individual oscillation during training in a year $(<6 \%)$ - this high stability of tHb- mass indicates that it could be included in an athlete's "biological passport" for monitoring against doping offences
}

Title: Total hemoglobin mass - A new parameter to detect blood doping? Authors: Prommer N, Sottas P-E, Schoch C, Schumacher YO, Schmidt W Reference: Med Sci Sports Exerc 2008; 40(12): 2112-2118 Type of study: Prospective study

Keywords: oping, blood, epo, hemoglobin, blood pass, intra-individual varianc

EB Rating: 7.5/10, Cl Rating: 7.5/10,

Background: number of doping measures have been used by athletes to increase total hemoglobin mass (tHbmass) but the normal variation of tHb-mass over a season in elite athletes has not been studied

Research question/s: What is the intra-individual variance of total hemoglobin mass (tHb-mass) in elite athletes during a training year at sea level?

\section{Methodology:}

- Subjects: 24 endurance athletes and 6 control athletes five times during a year and were compared with a control group $(n=6)$

- Experimental procedure: Total hemoglobin mass (tHb-mass) and hemoglobin concentration $([\mathrm{Hb}])$ were measured 5 times during a year in all the subjects. An analysis of covariance was then used to test the effects of training phases, age, gender, competition level, body mass, and training volume on the parameters.

- Measures of outcome: Measures on variation in the year were based on 1) a total \% error of measurement (TEM), 2) the combination of a typical \% error (TE) of analytical origin with an absolute SD of biological origin, and 3) between-subject and withinsubject variance components as obtained by an analysis of variance

\section{Main finding/s:}

- Total error of measurement (TEM): The Tem for tHb-mass was $2.2 \%$, and the effects of training volume $(p=0.20)$ and training phases $(p=0.81)$ on tHb-mass were not significant

- Variation: Within-subject variations had mainly an analytical origin (TE 1.4\%) and a very small biological origin

\section{Conclusion/s:}

- The measurement of total hemoglobin mass (tHb-mass) has a very low individual oscillation during training in a year $(<6 \%)$ - this high stability of $\mathrm{tHb}$-mass indicates that it could be included in an athlete's "biological passport" for monitoring against doping offences

\section{Methodological considerations:}

Well conducted study

SMU Volume 9 (2) 1 p4: 2009 Category: Medical / Haematology / Blood doping

The statements and opinions contained in the summaries of SportsMed Update are solely those of the individual authors and contributors and not of any organization or MPAH Medical cc. The information contained in summaries should never be used as a substitute for clinical judgment. The appearance of any promotional material in SportsMed Update is not a warranty, endorsement or approval of the products or services advertised or of their effectiveness, quality or safety. SportsMed Update, and the publisher, MPAH Medical cc, disclaims responsibility for any injury or illness to persons or dama to property resulting from any ideas or products red to in the summaries or advertisements.

Mit freundlicher Genehmigung der FIMS www.fims.org 